\title{
Binocular interactions in normal and anomalous binocular vision: effects of flicker
}

\author{
DENNIS M. LEVI, ANASTAS F. PASS, AND RUTH E. MANNY \\ From the University of Houston College of Optometry, 4901 Calhoun Blvd, Houston, Texas 77004, USA
}

SUMmaRY Temporal modulation thresholds were determined for monocular viewing and for binocular viewing of stimuli presented in phase or in counterphase to each eye of observers with normal binocular vision and those lacking stereopsis. The results showed that in individuals with normal binocular vision sensitivity was much greater for in-phase than for counterphase stimulation at low temporal frequencies, but that this superiority declined at higher temporal frequencies. Averaged across frequencies, binocular sensitivity for in-phase stimulation was $40-50 \%$ higher than monocular sensitivity. In contrast, in the observers lacking stereopsis the ratios of binocular in-phase/monocular sensitivity averaged $1 \cdot 02$, and there were no significant differences in sensitivity to in-phase and counterphase stimulation. This failure of binocular integration at threshold does not result from differences in transmission time between the 2 eyes. However, while individuals lacking stereopsis showed an absence of binocular interaction for uniform-field flicker at threshold, they showed suprathreshold dichoptic temporal frequency masking which was similar to that found in normal persons.

Sherrington's research on the integrative action of the central nervous system ' led him to hypothesise that, if the physiological activity of each eye was combined at a neural locus common to the 2 eyes, then perception of flicker during binocular viewing of in-phase light flashes should persist well beyond the fusion of flicker with monocular viewing, while a pattern of intermittent illumination presented in counterphase (180 degrees out of phase) to the 2 eyes would reduce or eliminate the perception of flicker. To test this hypothesis he compared the frequency at which flicker disappeared (CFF) when repetitive flashes were presented simultaneously (in phase) to the 2 eyes with the CFF obtained when the flashes were presented in counterphase to the 2 eyes. Sherrington found that the CFF for simultaneous flicker was only about $3 \%$ higher than the CFF for counterphase flicker, and concluded that no physiological interaction of consequence occurred between the signals from the 2 eyes. Several subsequent experiments have also shown small but significant differences between CFF for in-phase and counterphase stimulation. ${ }^{23}$

De Lange ${ }^{4}$ demonstrated that visual sensitivity to time-varying stimuli can be best determined by sinus-

Correspondence to Dr Dennis M. Levi. oidally modulating the brightness of a stimulus about a constant average level. By determining the depth of modulation required for the perception of flicker at different temporal frequencies he determined a temporal modulation sensitivity function (or De Lange function). Recent investigations of flicker sensitivity using the approach of De Lange have shown that, in the temporal contrast domain, when the stimuli to the 2 eyes were in phase, binocular sensitivity was greater than monocular sensitivity for lower temporal frequencies, but this difference in sensitivity decreased at higher frequencies. Moreover, when the stimuli were in counterphase, binocular sensitivity was less than monocular sensitivity at low frequencies but not at high frequencies. ${ }^{5-7}$ Thus, while Sherrington's hypothesis was correct, CFF may have been a rather insensitive measure of binocular interaction.

Persons lacking stereopsis due to early strabismus or amblyopia fail to show binocular summation on a variety of psychophysical threshold tasks. ${ }^{8-10}$ One possible explanation for the reduced or absent binocular summation is suppression. Suppression in strabismus is most marked when the 2 eyes are presented with similar contours ${ }^{11}{ }^{12}$ and requires a short latent period in order to become manifest. ${ }^{11}$ 
Clinically, brief exposures and/or rapid flashing of the stimuli are strategies commonly utilised to minimise or eliminate the suppression. ${ }^{113}$ Therefore it is likely that the temporal parameters of the stimuli presented to the 2 eyes might influence the degree of binocular summation found in persons with abnormal binocular vision. In the studies reported here we determined temporal modulation sensitivity functions for stimuli presented in phase and out of phase to the 2 eyes of persons with normal and abnormal binocular vision. This type of stimulus allows the determination of binocular interactions over a wide range of temporal frequencies and has the additional advantage of being less susceptible to differences in acuity or accommodation than patterned stimuli.

\section{Materials and methods}

Stimuli were generated on 2 matched cathode ray tube displays (Tektronix $5103 \mathrm{~N}$ ) which were viewed in a mirror stereoscope. The screens were masked to form a $6^{\circ}$ by $6^{\circ}$ square. A small square served as a fixation point in the centre of each screen, and horizontal and vertical nonius lines on the 2 screens were used to ensure that the stimuli were presented to corresponding points in the 2 eyes and that fusion was maintained throughout the experiments. Cover testing in the apparatus was used to further ensure appropriate binocular alignment.

The $\mathrm{Z}$ axis of each oscilloscope could be modulated in luminance sinusoidally about a mean luminance of $10 \mathrm{~cd} / \mathrm{m}^{2}$, which remained constant throughout the experiments.

\section{EXPERIMENTS}

Three separate experiments were conducted.

Experiment 1. Temporal modulation sensitivity: In phase versus counterphase.

In the first experiment temporal modulation sensitivity functions were measured for temporal frequencies from $1 \mathrm{~Hz}$ to $40 \mathrm{~Hz}$. At each temporal frequency the temporal contrast of the modulation was adjusted via a logarithmic attenuator. Thresholds were determined by an ascending method of limits, and for each contrast threshold the series of 5 measurements yielded a standard error of less than $5 \%$. Thresholds were determined for binocular stimulation in phase and $180^{\circ}$ out of phase (counterphase), and also monocularly, with the untested eye viewing a homogeneous field of the same mean luminance. The order of temporal frequencies and conditions was randomised. Trials in which one of the nonius lines was not seen, owing to suppression, were excluded from data analysis.

Experiment 2. The effect of small interocular delays.

Binocular temporal modulation sensitivity was determined for $1 \mathrm{~Hz}$ and $10 \mathrm{~Hz}$ flicker presented in phase to the 2 eyes, and with interocular phase lags of $15^{\circ}$ to $180^{\circ}$ in $15^{\circ}$ increments. The order of presentation of the stimuli was randomised, and monocular thresholds were also obtained for each eye during the same experimental session.

Experiment 3. Suprathreshold temporal frequency masking.

Temporal modulation sensitivity functions were determined monocularly by an ascending method of limits. At each temporal frequency the test stimulus was presented for 4 seconds. The observer was instructed to make a Yes/No response. Modulation was increased in $0.05 \mathrm{log}$ unit steps from below threshold until 2 consecutive positive responses were obtained. Threshold was taken as the first of 2 consecutive positive responses. Blank trials were included as a control for response bias. Five monocular thresholds were obtained at each temporal frequency under each of 2 conditions (1) while the untested eye viewed a blank field of the same average brightness, and (2) while the untested eye viewed a suprathreshold uniform field flicker 'mask' at 5,10 , or $20 \mathrm{~Hz}$, modulated about $1 \log$ unit above threshold.

\section{SUBJECTS}

Six observers between 20 and 30 years of age were tested. Two of the authors, each with normal binocular vision and stereopsis, served as normal participants. The other 4 observers all showed an absence of stereopsis (determined via the random dot E test, the Wirt stereotest, and the American Optical nearpoint vectograph). Two of the observers were classified as monofixators, and 2 had a small angle alternating esotropia. All had at least 20/30 or better acuity in the nondominant eye. All observers had clear media and normal fundi and were appropriately corrected for refractive error during the experiments.

\section{Results}

\section{EXPERIMENT 1}

Modulation sensitivity determined with in-phase (open circles) and counterphase (filled circles) flicker are shown for a normal observer in Fig. 1A. The ratio of in-phase to counterphase sensitivity at each frequency is shown by the circles below. At low frequencies $(<5 \mathrm{~Hz})$ this observer is considerably more sensitive to in-phase than to counterphase flicker, with the ratios decreasing monotonically at higher frequencies. The triangles in the same figure show the ratio of binocular sensitivity for in-phase flicker to the mean monocular sensitivity. For this normal observer binocular in-phase/monocular ratios averaged across frequencies was $1 \cdot 51$. This is similar to the $\sqrt{ } 2$ improvement for binocular viewing found 
NORMAL

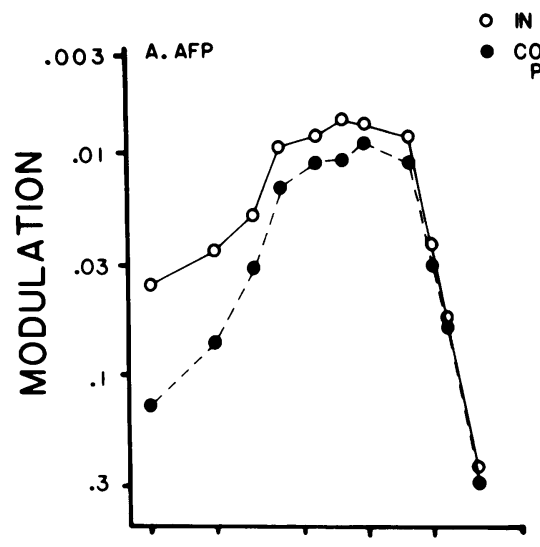

IN PHASE

COUNTER-
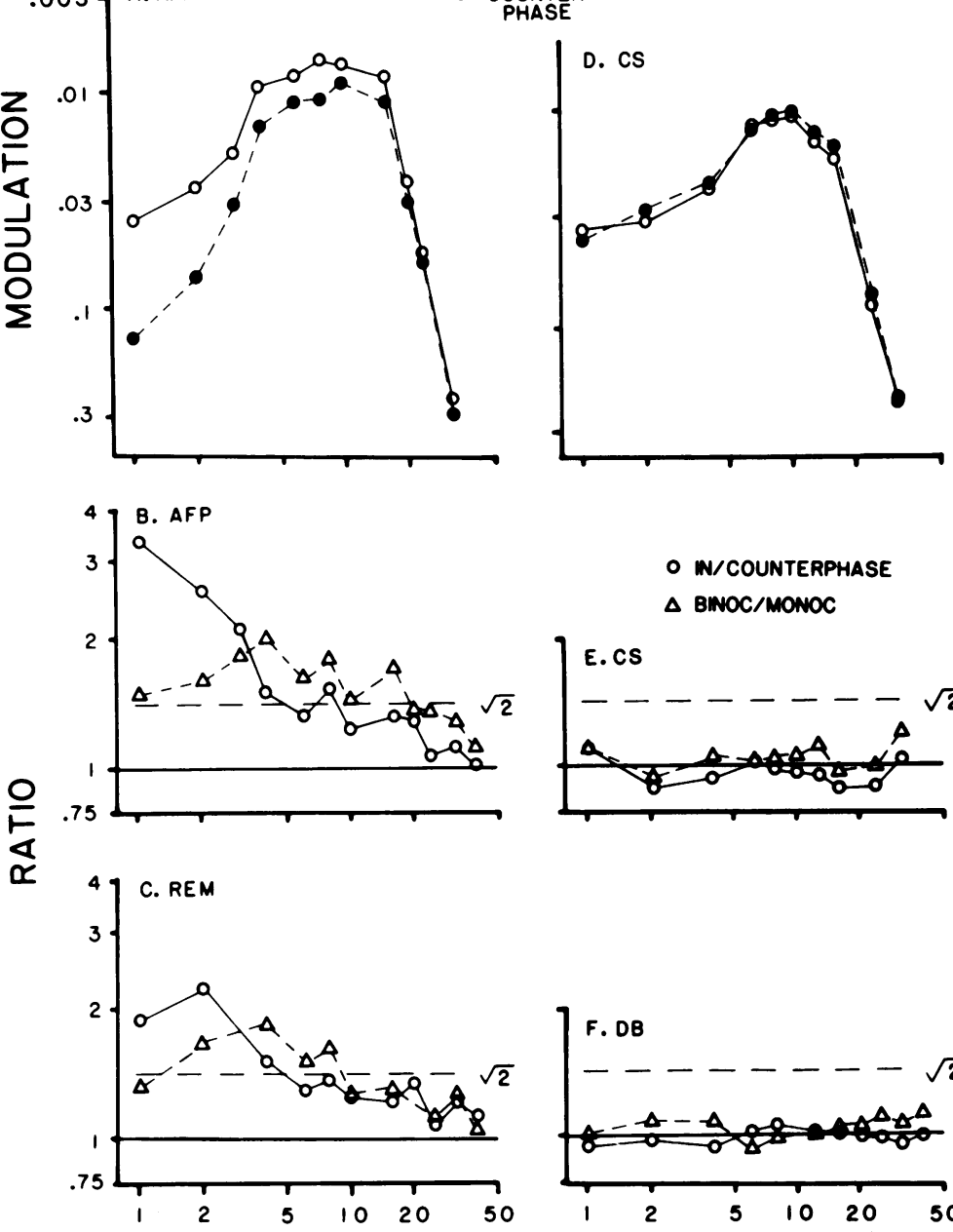

- W/COUNTERPHASE

STEREO BLIND

$\triangle$ BNOC/MONOC
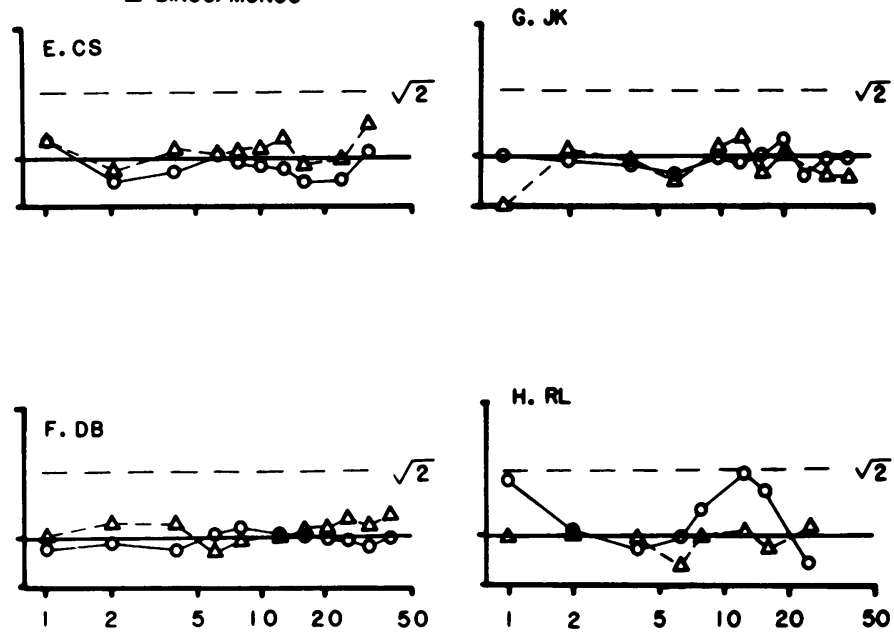

\section{TEMPORAL FREQUENCY}

Fig. 1 Modulation thresholds for binocular in-phase (open circles) and counterphase (solid circles) uniform field flicker for normal observer AFP (A) and for observer CS with anomalous binocular vision (D). The ratio plots below show the ratio of in-phase/counterphase sensitivity (circles) and the ratio of in-phase binocular/monocular sensitivity (triangles). The ratio plots on the left (B and $\mathrm{C})$ are for the normal observers; those on the right $(\mathrm{E}-\mathrm{H})$ are for the anomalous observers.

for spatial stimuli by Campbell and Green, ${ }^{14}$ a value which is predicted by assuming that the signals from the 2 eyes are summed and that the signals contain noise that is uncorrelated between the 2 eyes. This value is shown by the dashed line. It should be noted that the binocular in-phase/monocular ratio is also strongly dependent upon temporal frequency; thus the approximation to $\sqrt{ } 2$ may be somewhat misleading. Nevertheless the strong binocular summation, particularly at low temporal frequencies, is greater than would be predicted on the basis of probability and therefore must reflect neural summation. ${ }^{14}$ Similar results are shown for the other normal observer as a ratio plot in Fig. 1C.

The data reported thus far also suggest that in normal observers binocular interactions show strong temporal specificity. There is little difference between binocular in-phase sensitivity and either monocular or binocular counterphase sensitivity at high frequencies. However, at low frequencies there is 
considerable interaction between the 2 eyes, with binocular in-phase sensitivity enhanced, and counterphase sensitivity reduced as compared with monocular sensitivity. These results are in agreement with previous studies of normal binocular interactions. ${ }^{5-7}$

Data for an observer with anomalous binocular vision (CS) are shown in Fig. 1D. The sensitivity of this observer to both in-phase and counterphase flicker was quite similar, and did not differ significantly from the monocular sensitivity, as can be seen in the ratio plot below (Fig. 1E). Since the monocular thresholds for each eye of all of the observers were essentially equal, we have averaged the monocular thresholds for the 2 eyes. Essentially the same results were obtained by comparing the binocular thresholds with those of either the dominant or the nondominant eye. Similar results were obtained for the other observers with anomalous binocular vision, and their ratio plots are shown in Figs. 1F, G, and H. For the observers lacking stereopsis the binocular/monocular ratio averaged 1.02 , and the binocular in-phase/ counterphase ratios averaged across frequencies averaged 1.01 . For only 1 anomalous observer (RL, Fig. 1H) did the ratios of in-phase/counterphase stimulation exceed $1 \cdot 1$. This occurred at 2 distinct temporal frequencies, $1 \mathrm{~Hz}$ and $12.5 \mathrm{~Hz}$. Interestingly, at these 2 frequencies the monocular and binocular in-phase sensitivities were essentially equal. Thus these results may reflect inhibitory interactions at these 2 frequencies in the counterphase condition. However none of the observers with anomalous binocular vision showed significant binocular summation at any temporal frequency.

\section{EXPERIMENT 2}

One possible mechanism for the failure of observers without stereopsis to show binocular summation could be a difference in transmission time between the 2 eyes, so that the in-phase signals to the 2 eyes do not reach the brain simultaneously. Since no binocular summation was evident in the $180^{\circ}$ out-ofphase condition, we performed a second experiment in order to test more carefully the possibility that small interocular delays may preclude binocular summation. Thresholds were determined for $1 \mathrm{~Hz}$ and $10 \mathrm{~Hz}$ stimuli with different phase lags. This procedure allowed us to investigate the effects of delays as brief as $4 \mathrm{~ms}$. Fig. 2A shows the binocular/ monocular ratio for normal observer AFP for the 1 $\mathrm{Hz}$ (open symbols) and $10 \mathrm{~Hz}$ (filled symbols) stimuli. The abcissa shows the phase lag of the stimulus presented to the left eye with respect to that presented to the right eye. The lower 2 abcissas show the relative delay in milliseconds for the $10 \mathrm{~Hz}$ and $1 \mathrm{~Hz}$ stimuli. For this observer, when the stimuli were in phase, there was substantial binocular summation, which
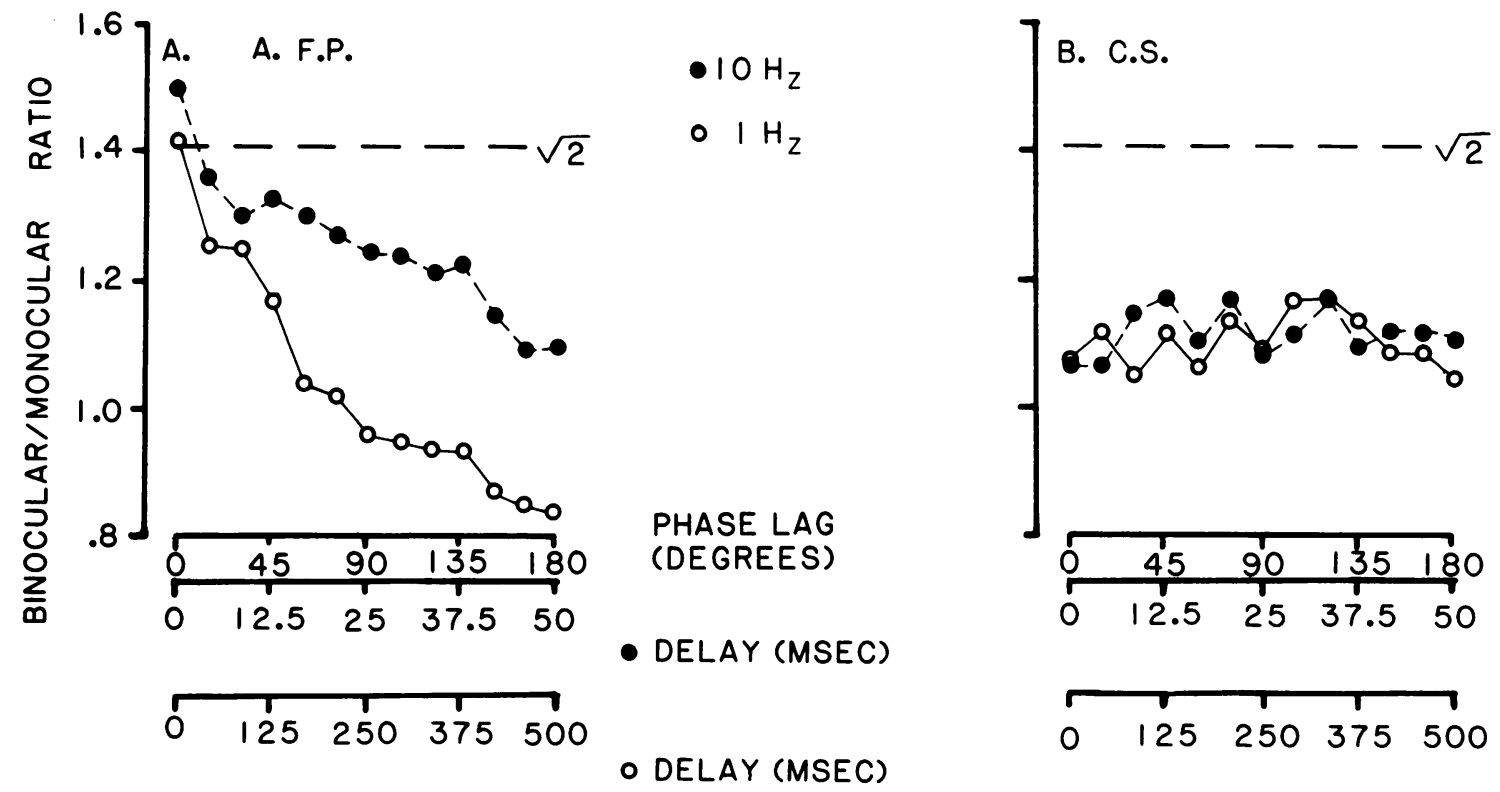

Fig. 2 The binocular/monocular sensitivity ratio as a function of phase for $1 \mathrm{~Hz}$ (open circles) and $10 \mathrm{~Hz}$ (solid circles) modulation for normal observer AFP (A) and anomalous observer CS (B). The abscissa shows the phase lag of the stimulus to the left eye with respect to that of the right eye. The lower 2 abscissas show the relative delay (in milliseconds) for the $10 \mathrm{~Hz}$ and $1 \mathrm{~Hz}$ stimuli. 
decreased as the phase lag between the 2 eyes increased. This decrease in binocular summation as a function of phase lag was more rapid at $1 \mathrm{~Hz}$ than at $10 \mathrm{~Hz}$, since the delay is 10 times greater at $1 \mathrm{~Hz}$ than at $10 \mathrm{~Hz}$ for a given phase lag. At $10 \mathrm{~Hz}$ the data fell to a binocular/monocular ratio of 1.1 with a $180^{\circ}$ phase lag. This $10 \%$ improvement of binocular performance might be predicted on the basis of probability summation. ${ }^{10}$ Interestingly, the $1 \mathrm{~Hz}$ data fall below 1.0 when the signals to the 2 eyes are more than $90^{\circ}$ out of phase, showing significant cancellation of the signals.

Data for an anomalous observer (CS) are shown in Fig. 2B. At both frequencies the binocular/monocular ratio hovered between 1.05 and 1.17 with an average of about $1 \cdot 1$. There was no delay which produced any significant binocular interaction, excitatory or inhibitory. Similar results were obtained by advancing the phase of the left eye signal with respect to the right eye. We should point out that while this observer showed a binocular/monocular summation ratio which was consistent with probability summation both at $1 \mathrm{~Hz}$ and $10 \mathrm{~Hz}$, this was not true for all temporal frequencies either for this observer or across observers as illustrated in the ratio plots of Fig. 1D-H. Most of the anomalous observers showed binocular/ monocular ratios which were closer to $1 \cdot 0$ over the range of frequencies tested. The data presented here are in agreement with studies of spatial contrast sensitivity in observers with abnormal binocular vision in showing an absence of binocular summation. ${ }^{89}$ The present results extend this absence of binocular summation into the temporal frequency domain, and further suggest that the absence of summation does not result from different transmission times between the 2 eyes.

\section{EXPERIMENT 3}

The results of experiments 1 and 2 indicate that anomalous observers lack excitatory binocular interactions at threshold. However, it has recently been reported that observers with abnormal binocular vision do show binocular interactions in suprathreshold dichoptic masking tasks. ${ }^{9}$ In these experiments a suprathreshold masking grating presented to one eye raised the contrast thresholds for gratings presented to the fellow eye within a narrow range of spatial frequencies and orientations centred about the spatial frequency and orientation of the mask. The results of the present experiment show that analogous binocular interactions occur in the temporal domain.

These results are shown for a normal observer (filled circles) and for a stereoblind observer (open circles) in Fig. 3. The results for each of the 3 masking frequencies are shown separately. In order to show the masking effect clearly the data are plotted as the ratio of unmasked to masked modulation sensitivity (i.e., the ratio of sensitivity when the untested eye viewed a blank field to that obtained when the untested eye viewed a suprathreshold uniform field flicker mask). The error bars on the right of the graphs show 2 standard deviations. For each masking frequency the flicker mask presented to the untested eye produced substantial elevation of the flicker thresholds of the tested eye. While the peak threshold elevation in each case coincided with the masking frequency, the masking is considerably broader than occurs in the spatial domain. Thus the $5 \mathrm{~Hz}$ mask has a width at half strength of more than 4 octaves. The 10 and $20 \mathrm{~Hz}$ masks appear to produce somewhat more peaked threshold elevation functions. What is of greater interest, however, is the similarity of these dichoptic temporal masking functions for the normal and stereoblind observers, in both magnitude and extent. Similar results were obtained regardless of which eye viewed the test and masking stimuli, and the data in Fig. 3 are representative of the functions obtained for the other normal observer and each of the observers with anomalous binocular vision.

\section{Discussion}

The results of these experiments suggest that persons with normal binocular vision show strong integration of the signals from the 2 eyes and that the nature of binocular interactions is highly dependent upon temporal frequency. These results are in agreement with several recent studies of binocular interactions for time-varying stimuli in normal persons. ${ }^{5-7}$ However, individuals lacking stereopsis fail to show significant binocular interactions at threshold for uniform field flicker at any frequency, even though these stimuli were equally detectable by each eye individually. This failure of binocular integration at threshold does not appear to result from differences in transmission time between the 2 eyes.

The present results are in agreement with previous reports suggesting that persons lacking stereopsis fail to show binocular summation at threshold..$^{8-10}$ The presence of suprathreshold dichoptic masking in the temporal domain, as well as in the spatial domain, ${ }^{9}$ suggests that these anomalous observers do retain binocular interactions which are similar to those found in normal persons. The broadly tuned dichoptic flicker masking found in these experiments may be analogous to the finding that adaptation to sinusoidal flicker produces a temporary elevation in the temporal contrast threshold. This adaptation effect is much less specific than that occurring for spatial stimuli, ${ }^{15}{ }^{16}$ though the effects of successive adaptation reportedly did not transfer interocularly. ${ }^{15}$ 
Fig. 3 Threshold elevation produced by the $20 \mathrm{~Hz}$ (top), $10 \mathrm{~Hz}$ (middle), and $5 \mathrm{~Hz}$ (bottom) temporal masks (i.e., ratio of log modulation sensitivity with a homogeneous background to that with the uniform field flicker background about 1 log unit above threshold) for normal observer REM (filled circles) and stereoblind observer CS (open circles). The error bar shows 2 standard deviations.

\section{Conclusions}

The present results, in conjunction with the recent reports that some stereoblind observers do show

\section{Normal}
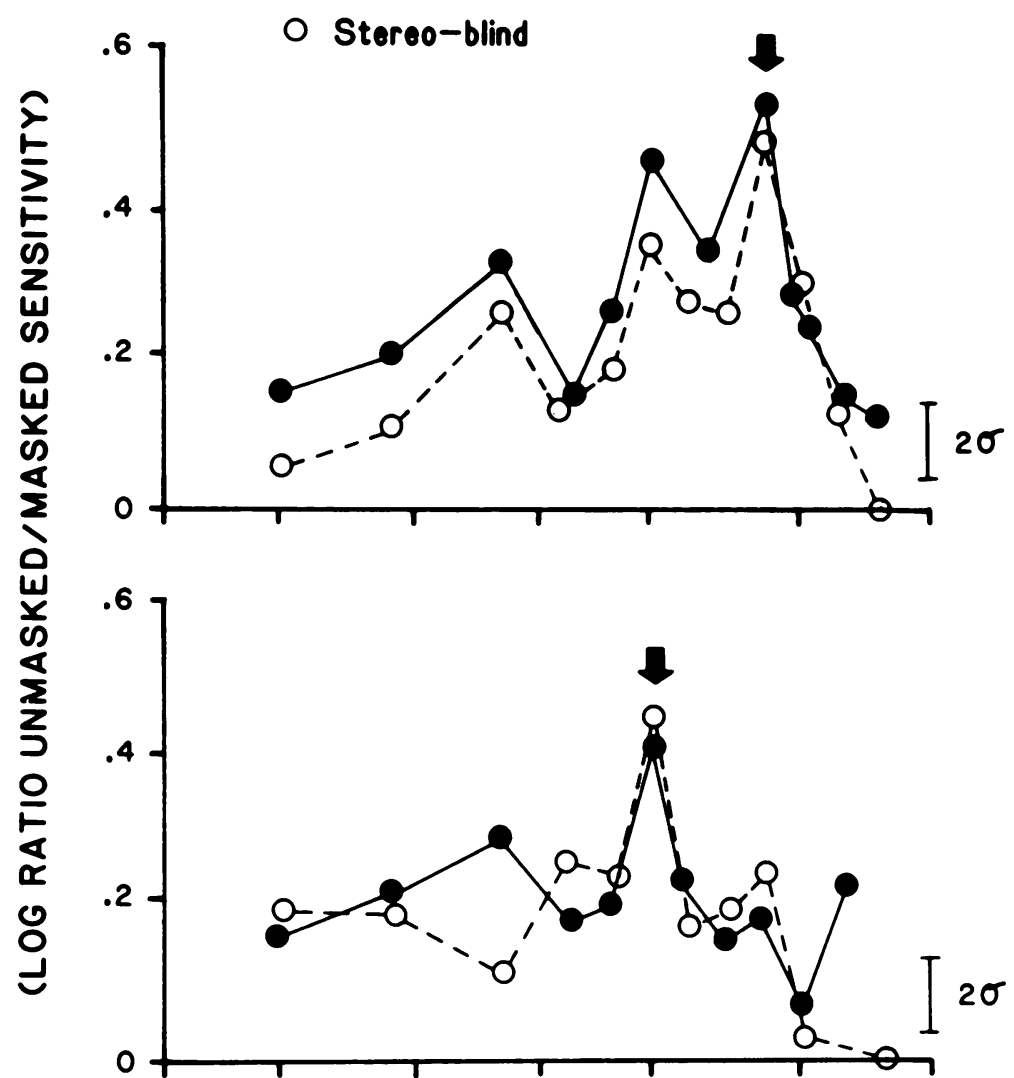

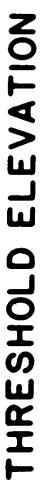

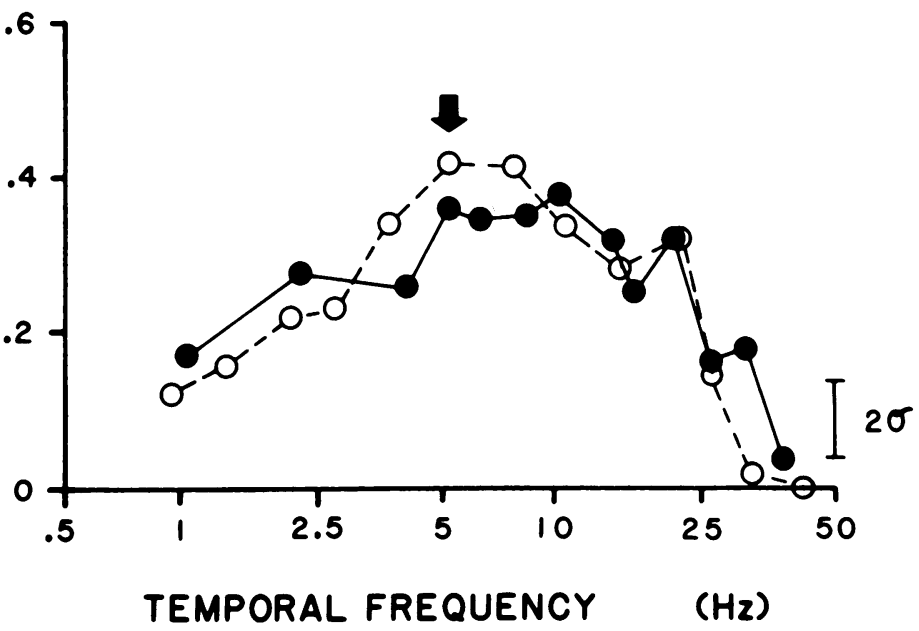

interocular transfer of the threshold elevation aftereffect ${ }^{17 i 8}$ and may show binocular interactions in the steady state visual evoked potential elicited by highcontrast gratings, ${ }^{19}$ suggest that some binocular 
neurons survive the disruptive effects of early abnormal binocular visual experience. It appears that the processes involved in these interactions are not as susceptible to the effects of early visual experience as the processes involved in binocular summation or stereopsis.

We thank Dr Ronald Harwerth for his valuable discussions and advice.

This work was supported by research grant RO1 EY 01728 from the National Eye Institute, Bethesda, Maryland, USA.

\section{References}

1 Sherrington CS. On binocular flicker and the correlation of activity of 'corresponding retinal points.' Br J Psychiatry 1904; 1: 26-60.

2 Ireland FH. A comparison of flicker frequencies under conditions of monocular and binocular stimulation. J Exp Psychol 1950; 40: 282-6.

3 Baker CH. A study of the Sherrington effect. Percept Psychophys 1970; 8: 406-10.

4 De Lange $\mathrm{H}$. Relationship between critical flicker frequency and a set of low-frequency characteristics of the eye. J Opt Soc Am $1954 ; 44: 380-9$.

5 Kintz TR. A comparison of monocular and binocular temporal resolution in human vision. University of Rochester, 1970: unpublished doctoral dissertation.

6 van der Tweel LH, Estevez O. Subjective and objective evaluation of flicker. In 4th Congr. Europ. Soc. Ophthal., Budapest, 1972. Ophthalmologica 1974; 169: 70-81.

7 Cavonious LR. Binocular interactions in flicker. QJ Exp Psychol 1979; 31: 273-80.

8 Lema SA, Blake R. Binocular summation in normal and stereoblind humans. Vision Res 1977; 17: 691-6.

9 Levi DM, Harwerth RS, Smith EL. Humans deprived of normal binocular vision have binocular interactions tuned to size and orientation. Science 1979; 206: 852-4.

10 Westendorf DH, Langston A, Chambers D, Allegretti C. Binocular detection by normal and stereoblind observers. Percept Psychophys 1978; 24: 290-14.

11 Jampolsky A. Characteristics of suppression in strabismus. Arch Ophthalmol 1955; 154: 683-96.

12 Schor CM. Visual stimuli for strabismic suppression. Perception 1979; 6: 583-93.

13 Griffin JR. Binocular anomalies: Procedures for Vision Therapy. Chicago: Professional Press, 1976: 264.

14 Campbell FW, Green DG. Monocular versus binocular visual acuity. Nature 1965; 208: 191.

15 Smith RA. Studies of temporal frequency adaptation in visual contrast sensitivity. J Physiol 1971; 216: 531-52.

16 Pantle A. Flicker adaptation-I. Effect on visual sensitivity to temporal fluctuations of light intensity. Vision Res 1971; 11: 943-52.

17 Hess R. Interocular transfer in individuals with strabismic amblyopia; a cautionary note. Perception 1978; 7: 201-5.

18 Anderson P, Mitchell DE, Timney B. Residual binocular interaction in stereoblind humans. Vision Res 1980; 20: 603-12.

19 Apkarian P, Levi D, Tyler CW. Binocular summation in the visual evoked potential of strabismic amblyopes. Am J Optom Physiol Opt 1981; 58: 820-30. 